\title{
Use of Genetic Algorithms for Optimal Design of Sandwich Panels Subjected to Underwater Shock Loading
}

\author{
Hamidreza Salimi1,* - Bahador Saranjam² - Ahmad Hoseini Fard ${ }^{1}$ - Mohsen Ahmadzadeh ${ }^{1}$ \\ 1 Shiraz Branch, Islamic Azad University, Shiraz, Iran \\ 2 Department of Marine Structures, Air Naval Research Center, Shiraz, Iran
}

Sandwich composite panels are increasingly used in the construction of marine vehicles because of their outstanding strength, stiffness and light weight properties. However, the use of composite panels comes with difficulties in the design process as a result of the large number of design variables involved, including composite material design, topologies and laminate schemes. Hence, this work deals with the presentation of an optimal design of laminated composite sandwich marine structures subjected to underwater explosion. The optimization process is performed using a genetic algorithm (GA), associated with the finite element method (FEM) for the structural analysis. In this optimization procedure, sandwich composite panel finite element model is built up, then the coupled acoustic-structural arithmetic from the widely used calculation program of the finite element "ABAQUS" is used to simulate and analyze the transient dynamic response of a sandwich composite panel that experiences loading by an acoustic pressure shock wave resulting from an underwater explosion "UNDEX". This approach is well suited for enhancing the response of orthotropic and/or laminated composites which involve many design variables. In GA method, a new approach is considered to improve this evolutionary algorithm for laminated stacking sequence and material selection of face layer and cores. Simple crossover, modified ply mutation, and a new operator called "ply swap" are applied to achieve these goals.

Keywords: optimization, genetic algorithm, finite element method, sandwich panel, underwater explosion, cavitation

\section{O INTRODUCTION}

Sandwich structures by definition are made of two thin faces with high stiffness and high strength and a core with low density and low stiffness. As an effective weight saving structure, sandwich structures were first applied in small airplanes during World War I.

Development of core materials has continued from the 1940's through today in an effort to reduce the weight. Nowadays, sandwich structures are used in almost every industrial sector ranging from buildings to aerospace applications because of the drive for lightweight structures with high stiffness to weight ratio, high bending strength to weight ratio, and good acoustical insulation [1]. Due to their high specific strength and high shock resistance signatures, composites are widely used as face materials in sandwich structures especially for large ship hulls. High stiffness combined with high energy absorption capability makes FGRP (Fiber Glass Reinforced Plastics) and CRP (Carbon Reinforced Plastics)faced sandwich structures ideal as the hull of marine vehicles. End-grain balsa wood and the closed-cell polymer foams such as polyvinyl chloride (PVC) or polyurethane are commonly used today as core materials in ship industry.

However, a large number of design variables and complex mechanical behavior associated with such materials turn the structural design into much more difficult and laborious than those involving conventional materials [2].
The earlier works in the field of composite structures optimization employed the same methods already used to optimize conventional material structures. These methods are based on gradients of the objective and constraints functions with respect to the design variables, which are considered to be continuous in the design space. Such works resulted in limited success because composite laminate design falls on a discrete optimization problem, since in practice the variables are restricted to few values imposed by the manufacturing process. Moreover, the composite optimization problems typically involve multimodal search spaces which may lead gradient based methods to converge to locally optimal regions in the design space [3]. Many other optimization techniques have been tested as an alternative to the gradient based methods, having the genetic algorithm (GA) stand out the others because it perfectly adjusts to the characteristics of the composite optimization problem. GAs are probabilistic search methods mimicking the biological reproduction and natural selection process through random but structured operators. The design variables usually restricted to discrete values are coded as genes using binary or integer numbers and grouped together in chromosomes strings that represent an organism (a possible solution in the design space). Instead of working with just one search point in the design space, GA uses a population of designs that by reproduction and selection operators evolve through successive generations. Many search points dispersed in the design space prevent the GA to get stuck in locally optimal regions, avoiding a 
premature convergence of the process. New designs are generated by the reproduction process that consists of the application of the genetic operators to parents selected from the existing population. These genetic operators are counterparts of the natural genetic mechanisms acting over the chromosomal strings of the organisms [4]. The selection of parents for the reproduction process and the selection of organisms to fill each new generation are both probabilistic. However, the chances of selection of each organism is proportional to its fitness, as it happens in the nature where fittest organisms have more chances to reproduce and to continue in the next generation. The organism fitness is obtained directly from an objective function using simple structure information and gradient evaluations are not required. Various researchers have studied the problem of sandwich panels optimization, but the use of GA and FEM together has not been widely explored, especially when the structure is subjected to underwater shock loading. In real designs cases, when the structural geometry is usually complex and the prediction of the structural behavior must be accurate, it is necessary to use numerical tools, such as the FEM, for the structural analysis.

In the present study, the optimal design of sandwich panels subjected to underwater shock loading is treated. The effect of cavitation on the structure is also considered. Cavitation is mentioned to a phenomenon which occurs in water, caused by the reflection of a shock wave at a free surface. For large structures, such as the design of a hull or superstructure, the optimization is divided into smaller, tractable, subproblems using predefined local loads to constrain the optimization [5]. The mass of the sandwich plates with orthotropic facesheets and core is minimized, considering deflection and certain failure loads as constraints. The design process requires the specification of the stacking sequence, which is defined by the orientation and material type of each ply layer, creating a discrete optimization problem.

Many researchers have proposed modifications to the classical GA structure to take advantage of composite laminate characteristics and minimize the computational cost. Some of these new strategies are applied in this work, consisting essentially of a GA restructuring of the variable codification and the genetic operators.

Given the superior strength-to-weight ratio, sandwich composite panels have been used extensively in the main structure of ships and underwater vehicles. In the present work an example of optimization of sandwich composite panels using parallel computing between the FEA and a developed genetic algorithm are studied. This approach is well suited for enhancing the response of orthotropic and/ or laminated composites which involve many design variables.

It is assumed that a sandwich panel consists of various facing lamination and different core thickness (Fig. 1). This plate structure is typical for the deck, side, and bottom of a ship hull girder. The loads acting on a panel in a ship is in-plane compression or tension, resulting from the overall hull-girder bending moment or torsion, shear force resulting from the hull-girder shear force, and lateral pressure resulting from the external wave or shock loading. Most studies of such structures generally consider shock loads in air.

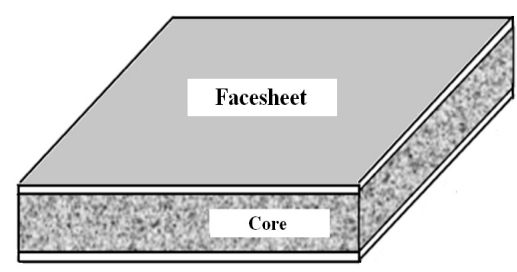

Fig. 1. Typical sandwich panel structure

This study attempts to utilize the finite element code "ABAQUS" [6] to examine the dynamic reaction of a sandwich panel to underwater shock loads. Underwater explosion pressure in that study is analyzed using Cole's formula [7]. Finally, the optimized stacking sequence of facesheets, the number of plies, fiber orientations and core thickness are determined by varying the ply angles and core thickness in order to achieve the minimum weight.

\section{APPROXIMATION OF SHOCK LOADING}

During an underwater explosion, the charge instantly converts the explosive energy into hot gas of approximately $3000{ }^{\circ} \mathrm{C}$ and induces a shock pressure of up to $5000 \mathrm{MPa}$ [7]. This investigation only considers the effects of the shockwaves. This shockwave propagates into the water medium with a spherical shape. The shock energy deliver to the structure produced by the underwater explosion is a function of the charge weight and the standoff distance. The shock pressure at a given point has a sharp peak in time, followed by a decaying exponential function, which is given by:

$$
P(t)=P_{\max } \cdot e^{-\left(\frac{t}{\lambda}\right)},
$$


where $P_{\max }$ is the peak pressure of the shock wave; $\lambda$ is the time decay constant, and $t$ is the time since the shock wave front arrived at the target point. For trinitrotoluene (TNT) or Pentolite, the peak pressure $P_{\max }$ and the time decay constant $\lambda$ are [7]:

$$
\begin{gathered}
P_{\max }=K_{1}\left(\frac{W^{1 / 3}}{R}\right)^{A_{1}}, \\
\theta=K_{2} \cdot W^{1 / 3} \cdot\left(\frac{W^{1 / 3}}{R}\right)^{A_{2}},
\end{gathered}
$$

where $W$ is the explosive weight, $R$ is the standoff distance, and $K_{1}, K_{2}, A_{1}$ and $A_{2}$ are the shock parameters of the explosive as defined in Table 1.

Table 1. Shock wave parameters [8]

\begin{tabular}{ccccc}
\hline Constants & $A_{1}$ & $A_{2}$ & $K_{1}$ & $K_{2}$ \\
\hline TNT & 1.18 & -0.185 & 52.12 & 0.092 \\
\hline
\end{tabular}

\section{SIMULATION AND ANALYSIS OF DYNAMIC RESPONSES OF A SANDWICH COMPOSITE PANELS}

In this work, the finite element code, "ABAQUS" [6], was applied to analyze the dynamic responses of sandwich composite panel subjected to an underwater explosion. ABAQUS consists of two main analysis products: ABAQUS/Standard and ABAQUS/ Explicit. ABAQUS/Standard is a general purpose analysis product that can solve linear and nonlinear problems involving the static, dynamic, thermal and electrical response of components. It solves a system of equations implicitly at each increment whereas ABAQUS/Explicit finds a solution forward through time in small time increments without solving a coupled system of equations at each increment [6].

ABAQUS/Explicit is a special purpose analysis product that uses an explicit dynamic finite element formulation. It is convenient for modeling transient dynamic events, such as blast, acoustic and shock problems. In ABAQUS, the shock analysis of a structure includes acoustic finite elements to model the effects of the mass of the fluid and incident wave loading to model UNDEX effects on the structure interacting with fluid. The explosive load is defined with an incident wave load. The load is applied on both the structure and the fluid at the common interface and is similar to a distributed load. These loads are supported only on transient dynamic procedures.

In Fig. 2, the shock loads acting on a sandwich panel in a ship arrangement is shown. The structural part is simulated by S4R four-node doubly curved shell element, shown in Fig. 3; and the infinite fluid domain was modeled and meshed using fluid 4-node AC3D4 acoustic tetrahedral elements in ABAQUS. The whole structural model surrounded by the fluid, as a FEM model, is depicted in Fig. 4. The fluid elements were given the properties of water. The bulk modulus of water was specified using the formula $\rho c^{2}$, where $\rho$ is the density of water and $c$ is the speed of sound in water.

The explicit time integration method is employed for computing time integration. Since the explicit time integration method is a conditional stable integration, the magnitude of the time step of the stable integration is a function of the element characteristic length. Therefore, when the mesh is divided into too small units, computational time is extended. In addition to considering integration stability, the division of the fluid element must account for the frequency of the shockwave, primarily because during transmission of the shockwave in the medium, the shockwave will be refracted and reflected when it runs into a gap or boundary, cause the shockwave to undergo superposition on or cancellation by the incident wave. To avoid this phenomenon in the computational process, which can result in significant errors, one must ensure that when analyzing a shockwave, each time step must not exceed that of two elements. Since the wavelength of a shockwave decreases as the frequency of a shockwave increases, this work can determine the minimum element length by calculating the upper range of shockwave frequency. The generalprinciple is $L_{\max }<c /\left(n \cdot f_{\max }\right)$, where $L_{\max }$ is the maximum permitted element length of the fluid element, $f_{\max }$ is the upper frequency range of the shockwave, $c$ is the acoustic speed of the fluid, and $n$ is the wavelength of the shockwave within the element, so the recommended value is $n \geq 6$ [6].

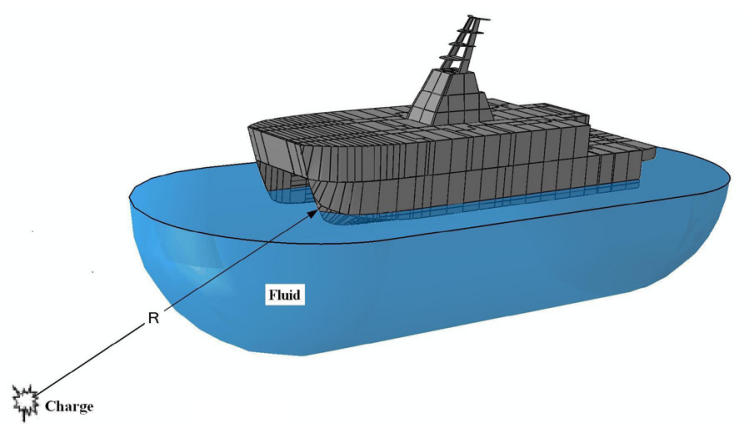

Fig. 2. Attack geometry of an underwater explosion

The distance between the explosion point and target panel is $7.62 \mathrm{~m}$, and the wavelength of the underwater shockwave is 6.7 to $10 \lambda$, as recommended 
by Keil [9]. Thus, the upper frequency range is $2665 \mathrm{~Hz}$. To ensure analytical accuracy, this study uses $n=9$; the length of the fluid element along the shockwave transmission direction is $60 \mathrm{~mm}$.

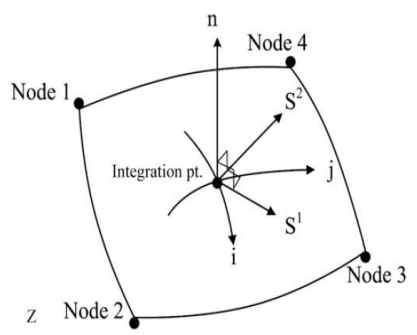

Fig. 3. The 4-node thin-shell element

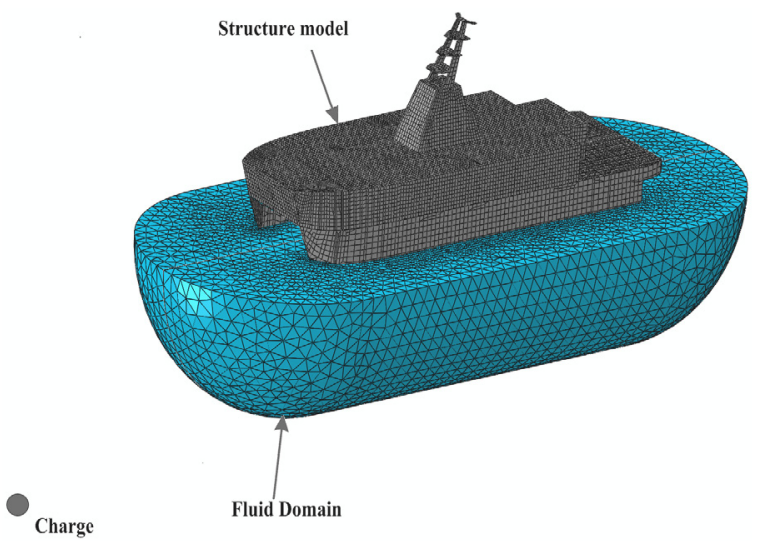

Fig. 4. Finite element models

The boundaries of the fluid may cause shockwave refraction or reflection, resulting in its superposition on or cancellation by the incident wave. To prevent this phenomenon, the boundary condition of the fluid element is set as a non-reflective boundary during the analysis. Restated, all pressure flows out of this boundary and will not cause reflection which typically affects the outcome of the analytical range. Between the sandwich panel and fluid, the nodal motion between the fluid and solid is connected by establishing the restraining condition of the interactive surface.

\section{OPTIMIZATION APPLICATION}

As discussed previously, the genetic algorithm is applied here to optimize a laminated composite sandwich panel which could not be performed appropriately by the gradient concept. GA is an optimization method that appeared in the early $70 \mathrm{~s}$. It is based on a simulation of Darwin's theory of species evolution. As such, GAs combine both exploration of the search space and exploitation of visited points.
However, contrary to classical optimization methods, GA does not provide an absolute optimum solution and the final result has to be obtained by inspection. The major strength of the GA method is multi-point discrete search technique ability, so it is possible to reach the global optimum.

\subsection{GA Details}

Typical GA elements include the encoding structure of the individual, operators to affect individuals (mutation, crossover and ply swap), a fitness criterion to determine the goodness of each individual and a selection function (selection). From one population GA then builds a new population with better global fitness to the criterion. Constraints in GA are handled in three ways: data structuring control repair operator and penalty functions. Data structuring control refers to defining the design variables, so that the optimizer always produces feasible designs. Since only the fittest individuals survive and reproduce, the genes of weaker individuals disappear gradually. Therefore, it follows if the environment (fitness law) does not change during the process, then finally the population will converge to a state where every individual has the fittest genes [10].

\subsubsection{Population}

There seems to be no novel idea by which to exactly decide the size of the population. This is especially true for complex problems. However, Goldberg et al. proposed an approximate population scaling law [11]. Biologically, individuals of the population make up a set of chromosomes which consist of combined genes and represent a solution set. In the present case, each ply must be encoded for the use in the GA. The values of plies are recorded in chromosomes as integer number encoding [3], For example, integer values from 0 to 2 represent the orientation of each ply. The positive integers map to orientation angles 0 to 90 and \pm 45 degrees from 1 to 2 , respectively, with the zero encoding representing an empty ply. In the present work chromosomes are composed of four parts (refer to Fig. 5).

\begin{tabular}{|c|c|cc|c|}
\hline+ & Orientation of each layer & Material type of each layer & $\uparrow$ \\
\hline $\begin{array}{c}\text { Number of layers } \\
\text { (1 gene) }\end{array}$ & (8 genes) & (8 genes) & $\begin{array}{c}\text { Core thickness } \\
(1 \text { gene })\end{array}$
\end{tabular}

Fig. 5. Chromosome structure

The distance between the explosion point and target panel is $7.62 \mathrm{~m}$, and the wavelength of the 
underwater shockwave is $6.7-10 \lambda$, as recommended by Keil [9]. Thus, the upper frequency range is $2665 \mathrm{~Hz}$. To ensure analytical accuracy, this study uses $n=9$; the length of the fluid element along the shockwave transmission direction is $60 \mathrm{~mm}$.

\subsubsection{Fitness}

Before making the random selection, each chromosome string is evaluated in the objective function relative to the chromosome's fitness. Stresses and deflections in each element are first calculated by FEA. Values of the safety factor for material failure is delivered from the FEA process to the GA process of Fig. 9 and used for fitness evaluation. The goal of the first step of the optimization is to find the lightest design for each panel that does not violate any of the imposed constraints. The problem is formulated as:

Minimize $W(\tilde{\tau})$ such that $G_{j}(\tilde{\tau}) \geq 0, j=1, \ldots$, $n_{g}$, where $W(\tilde{\tau})$ is the panel weight as a function of the design variables $\tilde{\tau}$, and $n_{g}$ is the number of constraints. The constraints are normalized, so that a constraint value of -0.1 corresponds to $10 \%$ constraint violation, while a constraint value of 0.1 corresponds to a $10 \%$ constraint margin. The design margin of safety is then defined by the most critical constraint $G_{\min }=\min \left(G_{j}\right)$. If $G_{\min }$ is negative, the design is infeasible, and a penalty is added to the objective function to help the search move into the feasible area of the design space. If $G_{\min }$ is positive, we have a feasible design with a positive margin, and we want to introduce a slight reduction to the objective function that will be a bonus for that margin.

\subsubsection{Selection}

Selection methods include roulette, ranking, tournament and elitist preserving [12]. The tournament selection approach is used here, with an elite's preservation. A random number generator in the GA plays an important role in the selection process of the various selection methods just cited. the tournament technique transfers the best fitness individuals among a certain number of selected individuals into the next generation by means of the random number. The process is repeated until the size of the population reaches the quorum. The elite preservation strategy prevents the best individual in a generation from being destroyed by mutation, crossover, and ply swap. The elite concept enables the best individual to always progress to the next generation [13].

\subsubsection{Crossover}

Crossover is an essential GA operator, having the fundamental task of creating new organisms (children) in a reproduction process. It acts by combining genetic information taken from a pair of organisms (parents) selected from the current population. The created children will hopefully be better than, or at least equivalent, in fitness to its parents. The crossover operator is applied by first generating a random number to define the crossover point. Then, the gene strings of both material and orientation chromosomes are split at the same point in both parents. For example, by splicing together the left part of the string of one parent with the right part of the string of the other parent, two child strings are generated (Fig. 6). The crossover operator is usually applied with some probability [14].

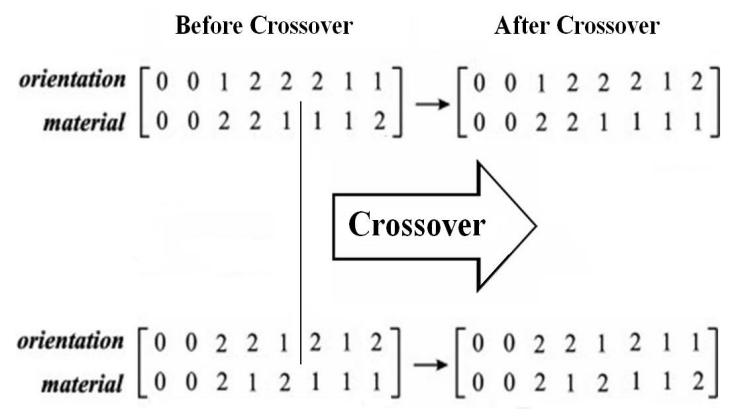

Fig. 6. Crossover operator

\subsubsection{Mutation}

After a child is created, the operators of adding, deleting, or mutating genes occur with small probabilities. These operators make up genetic mutation, and are illustrated in Fig. 7.

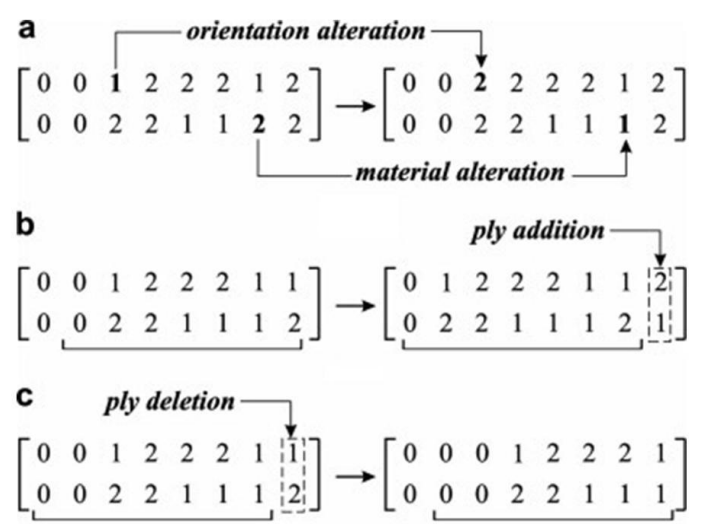

Fig. 7. Mutation

In order to avoid too rapid convergence of the population to a local optimum, the mutation function 
is used. For example, if the value of the first gene for all designs in the population achieved the same value, the mutation operator would be the primary means of introducing a different value to that bit; thus, mutation can help the crossover operator remain effective if the characteristics of all the designs in the population become uniform. When adding a ply stack, a uniform random number is chosen to the genes of the chromosomes. For the design problems considered in this work, outer plies in the laminate will get set up faster because they have a greater influence on the objective function, see Fig. 7a. To delete a ply stack, a random number is chosen and the corresponding stack is removed from the stacking sequence by replacing it with a 0 gene. The laminate is then re-stacked so that all empty plies are pushed to the outer edge of the laminate, see Fig. 7b. Gene alteration is shown in Fig. 7c. Each gene in the string switches with a small probability to any other permissible integer value.

\subsubsection{Ply Swap}

The ply swap operator is implemented by randomly selecting two genes in the string and switching their positions, see Fig. 8. The main characteristic of the ply swap operator is the ability to modify laminate stack sequence without changes of the total number of plies with fibers oriented on each permissible direction. Ply swap can be effective for problems where certain parts of the laminate stacking sequence get set up faster than others. For example, if the optimized stacking sequence for the outer section of the laminate has been determined first (as is the case for laminate design problems which involve bending), the ply swap operator may help the GA determine the optimized orientations for the inner part of the laminate by swapping plies from each section.

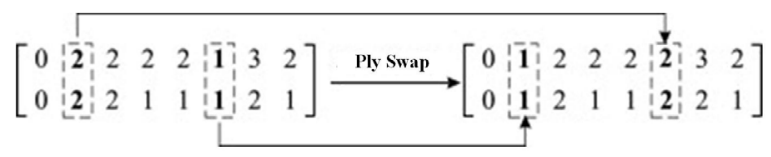

Fig. 8. Ply Swap operator

\subsubsection{Convergence Criteria}

It is known that GA does not lead to a unique solution and this is one of the major drawbacks of this technique. However, convergence does occur as fitness becomes better and this makes it possible to identify an appropriate time to terminate the GA. After the computation of several populations, the last one is then composed of several very good individuals according to the fitness criterion. Three convergence criteria are used in this work. If any of them is reached, then the optimization process terminates. These criteria are:

1. When the percentage difference between the average value of all the designs and the best design in a population reaches a very small specified value $c_{1}$,

$$
\frac{\left|W_{a}-W^{*}\right|}{\left|W_{a}\right|} \times 100 \leq c_{1},
$$

where $W^{*}$ is the fittest design in a population, $W_{a}$ is the average objective value in a generation defined by:

$$
W_{a}=\frac{1}{\mu} \sum_{j=1}^{\mu} W_{j}
$$

and $\mu$ is the population size,

2. If the fittest design has not changed for 50 successive generations, or the difference of the fittest design of the current generation, $W_{c}^{*}$, and that of 50 generations before, $W_{b}{ }^{*}$, is less than a small amount $c_{2}$, i.e.,

$$
\frac{\left|W_{c}^{*}-W_{b}^{*}\right|}{\left|W_{c}^{*}\right|} \times 100 \leq c_{2} \text {. }
$$

3. If the maximum number of generations is reached.

\subsection{Parallel Computing Technique}

Genetic Algorithm is very suitable for the parallel computing scheme because multiple design points should be evaluated in a calculation step. In other words, the algorithm can be programmed so that multiple design points in a generation may be divided into some sub-populations and the corresponding calculation of each sub-population is allocated to one processor in a parallel computer. The programming was coded with MPI (Message Passing Interface) library in this study. Its schematic diagram is shown in Fig. 9. The computing system used was CRAY-T3E and a PC cluster with 16 Pentium- 4 processors. The total CPU run time was approximately 3 days.

\section{OPTIMAL DESIGN OF COMPOSITE PLATES}

\subsection{Problem Definition}

The optimization problem can be formulated as finding the face sheet parameters (the number of 
layers, their materials, and ply angles) and the core thickness that satisfy strength and deflection constraints while minimizing the weight of the panel. Constraints considered include face sheets strength constraint, core transverse shear strength constraint, panel deflection constraint, and the symmetric layup constraint, but this is satisfied automatically by the coding rule that only half of the laminates are represented in a chromosome.

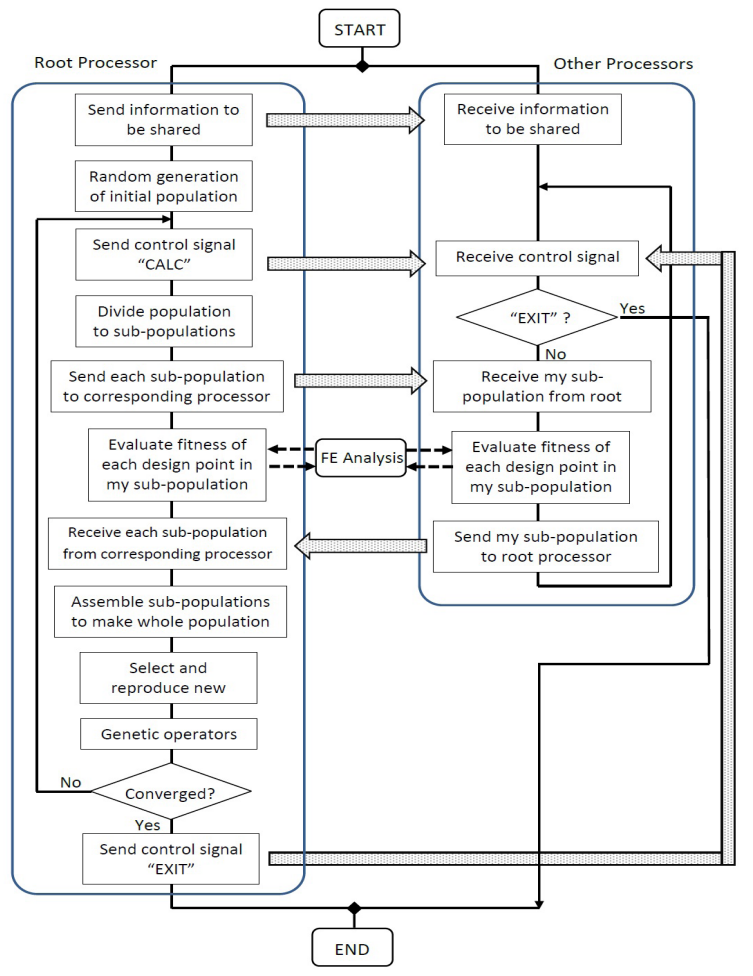

Fig. 9. Schematic diagram of Genetic Algorithm with parallel computing

The set of discrete (and commercially available) values of design variables is expressed as a vector $\tilde{\tau}$ $=\left\{\theta_{1}, \ldots, \theta_{n}, m_{1}, \ldots, m_{n}, t_{c}, n\right\}$, where $n$ is an implicit design variable dictated by the number of layers in the face sheet stacking sequence; $\theta_{i}$ and $m_{\mathrm{i}}$ are the orientation and material of the $i^{\text {th }}$ ply, respectively; $t_{c}$ is the core thickness. Material properties for the ply and the foam are provided in Table 2 and Table 3 , respectively. The design problem is typically formulated to provide a minimum mass structure:

$$
W=\left[t_{c} \rho_{c}+2 \sum_{i=1}^{n / 2} t_{f}^{i} \rho_{f}^{i}\right](a b)
$$

where $\rho_{c}$ and $\rho_{f}$ are material densities of the core and face ply, respectively; $n$ is the total number of plies; $a b$ is the panel area. The optimization problem with displacement and strength constraints can be expressed as $\min _{\tilde{\tau}} W(\tilde{\tau})$, such that;

$G_{c}(\tilde{\tau}) \geq 0 \quad$ (Compressive strength constraint),

$G_{t}(\tilde{\tau}) \geq 0 \quad$ (Tensile strength constraint),

$G_{c}(\tilde{\tau}) \geq 0 \quad$ (Shear strength constraint),

$G_{u}(\tilde{\tau}) \geq 0 \quad$ (Displacement constraint),

$t_{c} \in\{8,10,12\} \quad$ (Core thickness in $\mathrm{mm}$ ),

$\theta_{i} \in\left\{0^{\circ},-90^{\circ}, \pm 45^{\circ}\right\}, i=1, \ldots, n$ (ply angles),

$m_{i} \in\{$ Carbon/Polyester, Glass/Polyester $\}$

$n \in\left[n_{\min }, n_{\max }\right]$.

The critical constraint is defined as:

$$
G_{a}(\tilde{\tau})=\min \left\{G_{c}, G_{t}, G_{s}, G_{u}\right\},
$$

and the constrained optimization problem is transformed into an unconstrained maximization problem for the GA. This is done by using penalty parameters. The fitness function to be maximized is defined as:

$$
F(\tilde{\tau})=\left\{\begin{array}{cc}
-W(\tilde{\tau})+G_{c r} \delta, & G_{c r} \geq 0, \\
-W(\tilde{\tau})\left(1-G_{c r}\right)^{p}, & G_{c r}<0,
\end{array}\right.
$$

where $\delta$ and $p$ are bonus and penalty parameters, respectively.

Table 2. Material properties of the laminated facesheets

\begin{tabular}{lcc}
\hline \multicolumn{1}{c}{ Property } & $\begin{array}{c}\text { Glass } \\
\text { Polyester }\end{array}$ & $\begin{array}{c}\text { Carbon } \\
\text { Polyester }\end{array}$ \\
\hline Longitudinal modulus $\left(E_{11}\right)[\mathrm{GPa}]$ & 19.2 & 55 \\
Transverse modulus $\left(E_{12}\right)[\mathrm{GPa}]$ & 19.2 & 55 \\
In-plane shear modulus $\left(G_{12}\right)[\mathrm{GPa}]$ & 3.2 & 7.1 \\
Poisson's ratio $\left(v_{12}\right)$ & 0.32 & 0.3 \\
Density $(\rho)\left[\mathrm{kg} / \mathrm{m}^{3}\right]$ & 1619 & 1500 \\
Longitudinal tensile Strength $\left(X_{T}\right)[\mathrm{MPa}]$ & 227 & 350 \\
Longitudinal compressive Strength $\left(X_{C}\right)[\mathrm{MPa}]$ & 150 & 280 \\
Transverse tensile Strength $\left(\mathrm{Y}_{T}\right)[\mathrm{MPa}]$ & 227 & 350 \\
Transverse compressive Strength $\left(Y_{C}\right)[\mathrm{MPa}]$ & 150 & 280 \\
In-plane shear strength $(S)[\mathrm{MPa}]$ & 35 & 70 \\
Thickness $\left(t_{f}\right)[\mathrm{mm}]$ & 0.7 & 0.5 \\
\hline
\end{tabular}

\section{RESULTS AND DISCUSSION}

The optimum weight of the laminate is obtained in terms of thickness of plies, stacking sequence, number of plies etc., for a given underwater explosion induced by $18.3 \mathrm{~kg}$ TNT fired $7.62 \mathrm{~m}$ away from the bow of the hull. Therefore, the spherical incident wave is applied as a transient load active on both the acoustic and structural meshes at their common surfaces (the wetted interface). The geometric shape of the sandwich panel in a ship arrangement is shown in Fig. 10. 
Table 3. Material properties of the core

\begin{tabular}{lc}
\hline \multicolumn{1}{c}{ Material properties } & H-250 PVC \\
\hline In-plane tensile modulus [GPa] & 0.2898 \\
Transverse tensile modulus [GPa] & 0.2898 \\
In-plane tensile strength [MPa] & 6.1824 \\
Transverse tensile strength [MPa] & 8.5008 \\
In-plane compressive modulus [GPa] & 0.1449 \\
Transverse compressive modulus [GPa] & 0.3864 \\
In-plane compressive modulus [MPa] & 5.1198 \\
Transverse compressive strength [MPa] & 5.6028 \\
Transverse shear modulus [GPa] & 0.104328 \\
Transverse shear strength [MPa] & 4.347 \\
Density [kg/m³] & 252.8842 \\
Poisson's ratio & 0.32 \\
\hline
\end{tabular}

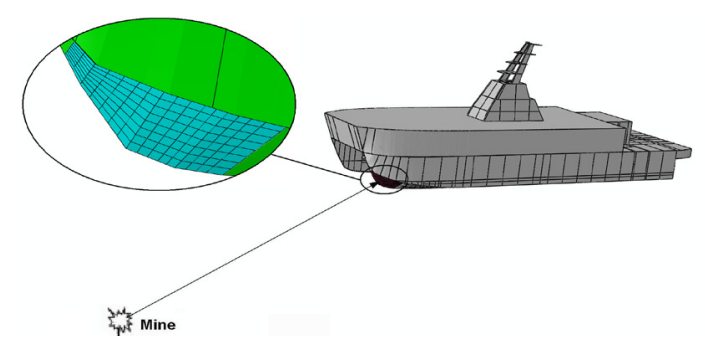

Fig. 10. Sandwich panel in this study

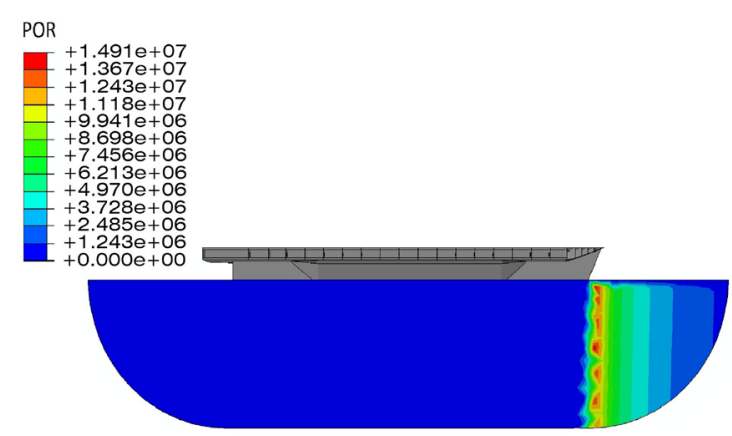

Fig. 11. Shock wave propagation

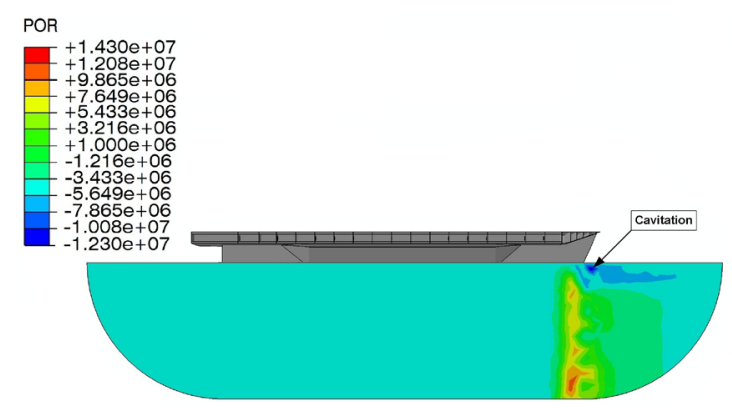

Fig. 12. Cavitation zone

The shock wave propagation is shown in Fig. 11. The maximum shock wave was calculated as $1.491 \times 10^{7} \mathrm{~Pa}$ which is close to the theoretical value of $1.488 \times 10^{7} \mathrm{~Pa}$ based in Eq. (2).
When the pressure is lower than the fluid vapor pressure, local cavitation effects appear between the fluid and structure which causes the target to separate from the fluid. This local cavitation is depicted in Fig. 12; blue colors specify the zone and distribution of local cavitation formed on the fluid-solid interface after the shockwave hit the structure.

The parameters used for the GA are shown in Table 4. By parametric study, the probabilities of mutation, crossover and ply swap were selected as $0.1,0.75$ and 0.05 , respectively. With a population size of 30 , iterations more than 50 were sufficient for convergence.

Table 4. Parameters of GA

\begin{tabular}{lc}
\hline \multicolumn{1}{c}{ Parameters } & Value \\
\hline Chromosome length & 18 \\
Upper limit of generation & 100 \\
Population size & 30 \\
Probability of mutation & 0.1 \\
Probability of crossover & 0.75 \\
Probability of ply swap & 0.05 \\
\hline
\end{tabular}

Table 5. Optimized materials and core thickness

\begin{tabular}{cccc}
\hline & Facesheets materials & $t_{c}[\mathrm{~mm}]$ & $W[\mathrm{~kg}]$ \\
\hline 1st Best $^{\text {nd Best }}$ & {$[\mathrm{C} / \mathrm{G} / \mathrm{C} / \mathrm{C} / \mathrm{G} / \mathrm{G}]_{\mathrm{S}}$} & 12 & 113.18 \\
$2^{\text {nd }}$ & {$[\mathrm{G} / \mathrm{C} / \mathrm{G} / \mathrm{C} / \mathrm{G} / \mathrm{G}]_{S}$} & 12 & 118.93 \\
$3^{\text {rd Best }}$ & {$[\mathrm{G} / \mathrm{C} / \mathrm{C} / \mathrm{G} / \mathrm{C} / \mathrm{G} / \mathrm{G}]_{\mathrm{S}}$} & 10 & 120.65 \\
\hline
\end{tabular}

Table 6. Optimized facesheets orientations

\begin{tabular}{ccc}
\hline & \multicolumn{1}{c}{ Facesheets orientations } & $W[\mathrm{~kg}]$ \\
\hline 1st Best & {$[0-90 / 0-90 / \pm 45 / 0-90 / \pm 45 / \pm 45]_{S}$} & 113.18 \\
2nd Best & {$[0-90 / 0-90 / \pm 45 / \pm 45 / 0-90 / \pm 45]_{S}$} & 118.93 \\
3rd Best & {$[ \pm 45 / 0-90 / 0-90 / \pm 45 / 0-90 / \pm 45 / \pm 45]_{S}$} & 120.65 \\
\hline
\end{tabular}

The possible maximum number of plies was set as 16 and only 8 plies were used as the design variables because all laminates were assumed to be symmetric. The symmetric laminate may have any number of plies and each ply may be made of either Glass/Polyester or Carbon/ Polyester (refer to Table 2 to compare the properties). The usable ply angles were limited to 0 to $90^{\circ}$ and $\pm 45^{\circ}$ for the practical application. The allowed thickness of the core is 8,10 or $12 \mathrm{~mm}$ and made of Divinycell HD-250. Material properties for the core are provided in Table 3. To start the optimization, the base model is built in "ABAQUS" version 6.10. Then, the "ABAQUS" Input File (.inp) is generated. This .inp file is modified with design variables of each chromosome string. To calculate the fitness value of each member of the population, "ABAQUS" runs the input file. Fig. 13 shows the convergence of fitness 
with the number of generations for four different initial populations. It could be observed that about 50 generations are required for convergence. Also, all of the four diagrams converge to the unique value. The GA stopping condition is either a limit on the total number of function evaluations or when there is no change in the fitness function between generations, whichever occurs first. Results for three best stacking sequences are shown in Tables 5 and 6.

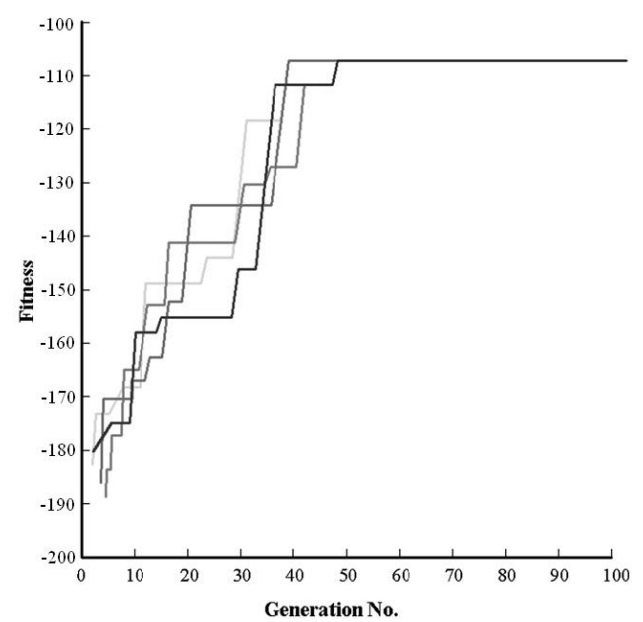

Fig. 13. Convergence plot for weight minimization with four different initial populations

\section{CONCLUSIONS}

A technique for combining genetic algorithms with the finite element method to minimize the weight of sandwich panel with laminated composite facesheets with several design variables is described in this paper.

The GA was successfully applied to obtain the optimal design of sandwich panels. It has been shown that the number of plies, stacking sequence of facesheets, fiber orientations and core thickness could be improved considerably by optimization process, which was demonstrated by a comparison of the design constraints between initial and optimized designs. The performance of the GA in optimized design of sandwich panels was studied, showing that the method is very efficient in finding near optimal solutions, and an important saving in computer time can be obtained by using of suitable values for the GA parameters and when results of different analyses are stored.

The resultant design with reasonable values of design variables proves that the optimized values of the design variables are even difficult to guess for a skillful engineer with exceptional experience. The results demonstrated that when relatively small populations associated with a large limit of the number of generations are used, better performances of the GA are obtained.

\section{REFERENCES}

[1] Zenkert, D. (1997). The Handbook of Sandwich Construction. Chameleon Press, London.

[2] Goubalt, P., Mayes, S. (1996). Comparative analysis of metal and composite materials for the primary structures of a patrol craft. Naval Engineers Journal, vol. 108, no. 3, p. 387-394, DOI:10.1111/j.1559-3584.1996. tb01575.x.

[3] Soremekun, G.A.E., Gürdal, Z., Haftka, R.T., Watson, L.T. (2001). Composite laminate design optimization by genetic algorithm with generalized elitist selection. Computers and Structures, vol. 79, p. 131-143, DOI:10.1016/S0045-7949(00)00125-5.

[4] Goldberg, D.E. (1989). Genetic algorithms in search, optimization, and machine learning. Addison-Wesley Publishing Company, Boston.

[5] McMahon, M.T. Watson, L.T. (2000). A distributed genetic algorithm with migration for the design of composite laminate structures. Parallel Algorithms and Applications, vol. 14, p. 329-362, DOI:10.1080/10637199808947394.

[6] ABAQUS User's Manual, Version 6.10 (2010). Hibbitt, Karlsson \& Sorensen, Inc., Michigan.

[7] Cole, R.H. (1965). Underwater explosions. Dover Pub. Inc., New York.

[8] Rajendran, R. (2009). Numerical simulation of response of plane plates subjected to uniform primary shock loading of non-contact underwater explosion. Materials and Design, vol. 30, no. 4, p. 1000-1007, DOI:10.1016/j.matdes.2008.06.054.

[9] Keil, A.H. (1961). The response of ships to underwater explosions. Transaction of Society of Naval Architecture and Marine Engineering, vol. 69, p. 366-410.

[10] Eiben, A.E., Smith, J.E. (2007). Introduction to Evolutionary Computing, 2nd ed. Springer, Berlin.

[11] Goldberg, D.E., Deb, K., Clark, J.H. (1992). Genetic algorithms, noise, and the sizing of populations. Complex Systems, vol. 6, no. 4, p. 333-362.

[12] Gen, M., Cheng, R. (2000). Genetic algorithms and engineering optimization. Wiley-Interscience, New York.

[13] Michalewicz, Z. (1996). Genetic algorithms + data structures $=$ evolution. Springer, New York.

[14] Gürdal, Z., Haftksa, R.T., Hajela, P. (1998). Design and optimization of laminated composite materials. WileyInterscience, New York. 